\title{
Logistische Materialvorhaltung der Polytraumaversorgung im Schockraum aus unfallchirurgischer Sicht unter Berücksichtigung des Status der Klinik: überregionales Traumazentrum Universitätsklinikum Augsburg
}

\author{
Michael Ecker
}

\begin{abstract}
Polytraumaversorgung 2019
Die Polytraumaversorgung in der Bundesrepublik Deutschland wurde mit der Publikation der 1. Auflage des „Weißbuchs Schwerverletztenversorgung“ von der Deutschen Gesellschaft für Unfallchirurgie (DGU) im Jahr 2006 neu strukturiert. Das Weißbuch enthält Empfehlungen zur Struktur, Organisation und Ausstattung der Schwerverletztenversorgung in der Bundesrepublik Deutschland und wurde bereits 2012 nach den Erfahrungen innerhalb des TraumaNetzwerks der Deutschen Gesellschaft für Unfallchirurgie und unter Berücksichtigung der aktuellen S3-Leitlinie Polytrauma überarbeitet. Damit konnte sichergestellt werden, dass die am TraumaNetzwerk (TNW) der DGU teilnehmenden und zertifizierten Kliniken deutschlandweit die medizinische Versorgung schwerstverletzter Patienten nach aktuellem Wissensstand der Medizin auf allerhöchstem Niveau durchführen. Zielsetzung des TNW ist die Bildung eines strukturierten flächendeckenden Verbundes von qualifizierten Kliniken zur Schwerverletztenversorgung nach einheitlichen Versorgungs- und Qualitätsstandards. So sollen für jeden Schwerverletzten an jedem Ort in Deutschland zu jeder Zeit die gleichen Überlebenschancen sichergestellt werden [1].
\end{abstract}

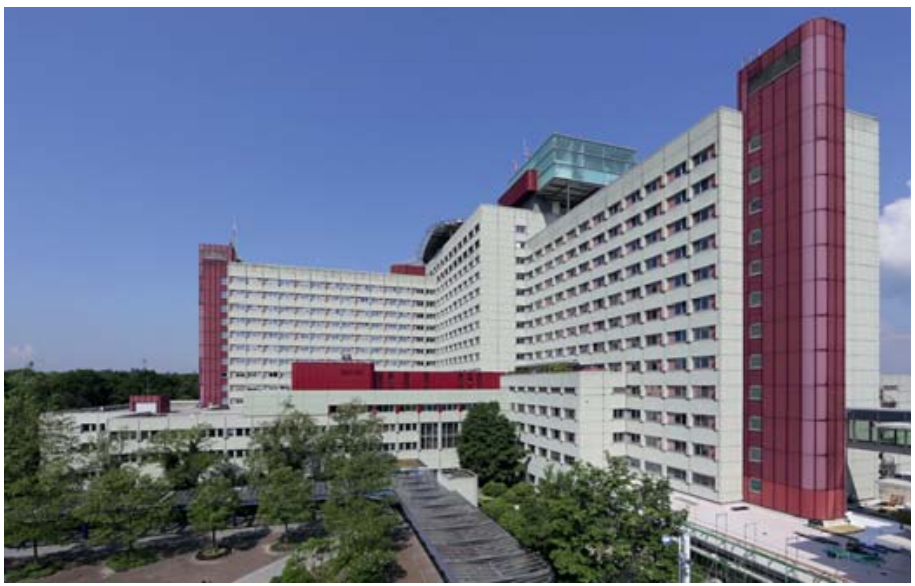

- Abb. 1 Universitätsklinikum Augsburg. Quelle: Universitätsklinikum Augsburg, Ulrich Wirth (2019).
Je nach Größe und Leistungsfähigkeit der einzelnen Klinik gibt es lokale, regionale und überregionale Traumazentren.

\section{Polytraumaversorgung am Universitätsklinikum Augsburg}

Das Universitätsklinikum Augsburg mit seinen über 1700 Betten ist zertifiziertes überregionales Traumazentrum mit allen Fachabteilungen und Haus der Maximalversorgung. Aufgrund der geografischen Lage des Großklinikums sowie der lokalen Krankenhausstruktur in der Region werden im Universitätsklinikum Augsburg, gemessen an der Bevölkerungsdichte, überdurchschnittlich viele Notfallpatienten behandelt. Jährlich werden in der Notaufnahme des Großkrankenhauses ca. 80000 Patienten behandelt, davon ca. 25000 unfallchirurgisch-orthopädische Patienten. Über 200 schwerstverletzte Patienten mit einem ISS (Injury Severity Score) $\geq 16$ wurden 2018 in den Schockräumen des Universitätsklinikums Augsburg ( $\triangleright$ Abb. 1) behandelt. Die Gesamtzahl der im Schockraum behandelten traumatologischen Patienten lag bei 879 [2].

\section{Schockraumalgorithmus}

Um einen reibungslosen Ablauf bei der Behandlung von Schwerstverletzten gewährleisten zu können, muss ein detaillierter Algorithmus erarbeitet werden [3]. Der sog. Schockraumalgorithmus im Universitätsklinikum Augsburg wurde anlässlich der Teilnahme am TNW und im Rahmen der ersten Zertifizierung als überregionales Traumazentrum komplett überarbeitet. Hierzu war es notwendig, im Rahmen einer Arbeitsgruppe, bestehend aus Mitarbeitern aller an der Schockraumversorgung beteiligten Kliniken und Berufsgruppen, die einzelnen Phasen von der Präklinik bis zur Verlegung des Schockraumpatienten in den OP oder auf Intensivstation gemäß den Vorgaben des Weißbuchs und unter Berücksichtigung der AWMF-S3-Leitlinie Polytrauma sämtliche Phasen und Schritte klar zu definieren. Der Schockraumalgorithmus ( $\triangleright$ Abb. 2) wurde hinsichtlich der einzelnen Behandlungsphasen streng unter Berücksichtigung des ATLS (Advanced Trauma Life Support ${ }^{\circledR}$ ) erstellt. 


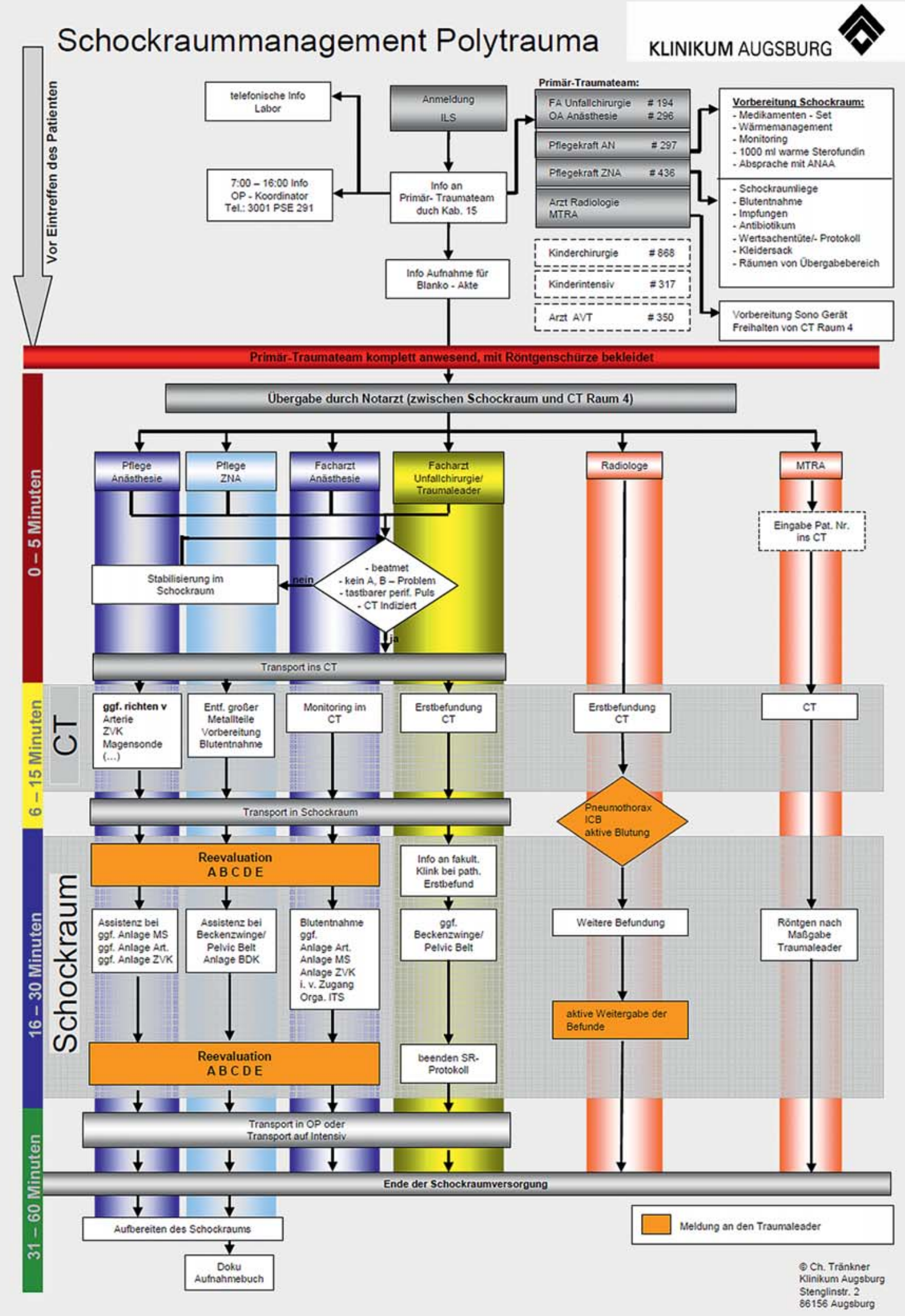

- Abb. 2 Schockraumalgorithmus. Quelle: Ch. Tränkner, Augsburg. 


\section{Schockraumindikationsliste}

Sturz aus mehr als 3 Metern Höhe

Explosionsverletzungen

Einklemmung/Verschüttung

Herausschleudern aus dem Fahrzeug

Tod eines Mitfahrers

Motorrad- oder Autounfall mit höherer Geschwindigkeit

als Fußgänger oder Radfahrer angefahren

Unfälle mit Pferden und Rindern

(Reitunfälle, Hornverletzungen, u. Ä.)

$\square$ nicht erweckbarer (Alkohol-)Intoxikierter mit Kopfverletzung

Schwerstkranke mit mutmaßlich akutem operativem und/oder anästhesiologischem Handlungsbedarf

(z.B. akute respiratorische und hämodynamische Notfälle; V.a. rupturiertes Aneurysma oder Dissektion der Aorta oder anderer großer Arterien; hämorrhagische Schockzustände, nicht selten aus den Fachgebieten Gynäkologie, Geburtshilfe, HNO, Urologie u. a.; V.a. SAB; V.a. Basilaristhrombose; V.a. hypertensive zerebrale Massenblutung)

\section{Anforderung des Schockraumteams durch den Notarzt}

- Abb. 3 Schockraumindikationsliste.

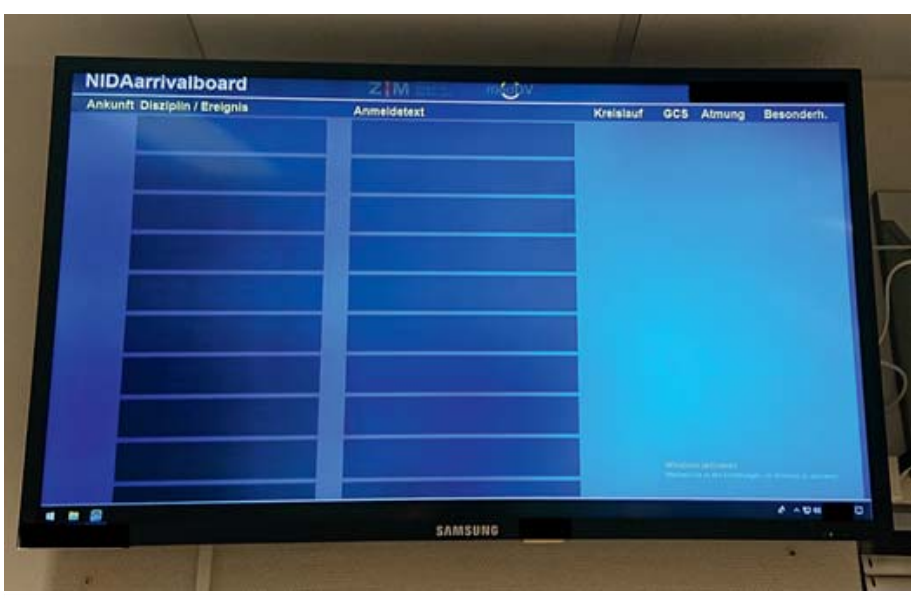

Abb. 4 Klinikmonitor.
Die Bedeutung des ATLS im Schockraum hinsichtlich der Verbesserung der Behandlungsergebnisse ist unumstritten und konnte in mehreren Studien herausgearbeitet werden [4-6].

Unserer Erfahrung nach ist eine gute und klare Kommunikation zwischen Schockraumteam und den Kräften vor Ort (Präklinik) mit ein Baustein zur Verbesserung der Polytraumaversorgung. Die für eine Klinik zuständige Leitstelle muss in den Schockraumalgorithmus integriert werden. So sichert eine optimale Kommunikation zwischen Präklinik und Schockraumteam einen zügigen und vollständigen Informationsaustausch [6].

Gemeinsam mit dem für die Integrierte Leitstelle Augsburg (ILS-Augsburg) zuständigen Ärztlichen Leiter Rettungsdienst wurde der Schockraumalgorithmus sowie die

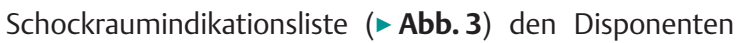
der ILS-Augsburg erläutert. Die Notarztstandorte sowie die RTH/ITH-Stationen wurden ebenfalls informiert.

\section{Patientenanmeldung im Traumazentrum}

Durch die immer knapper werdenden Ressourcen in Krankenhäusern durch Zunahme der Patientenzahlen bei gleich bleibender Personalstärke, die Bemühungen nach Effizienzsteigerung sowie das landesweite Streben nach Ökonomisierung der Krankenhäuser [7] sind alle an der Polytraumaversorgung beteiligten Kliniken einem extrem hohen Leistungsstress ausgesetzt. Die hohe Personalbindung bei der Versorgung Schwerstverletzter geht meist zulasten anderer Bereiche der Klinik. So kommt es durch einen Schockraumeinsatz oftmals zu einem Stopp in einem Operationssaal, um entsprechendes Personal für den Schwerstverletzten vorhalten zu können. Um unnötige Wartezeiten auf den Schwerverletzten mit hoher Personalbindung zu vermeiden und die Zahl der Schockraumeinsätze für leicht verletzte Patienten zu reduzieren, muss die aufnehmende Klinik exakte Informationen von der Einsatzstelle über die Leitstellen bekommen. Das Universitätsklinikum Augsburg verfügt seit 4 Jahren über ein digitales Voranmeldesystem. Die Rettungskräfte vor Ort haben die Möglichkeit, den Patienten online in Echtzeit in der Klinik anzumelden. Die IT-Lösung „NIDA“ vernetzt Rettungswagen und Kliniken, Ärzte können Notfälle dadurch schneller behandeln [8].

Auf einem Monitor in der Klinik ( $\triangleright$ Abb. 4 und 5) erscheinen die realen Patientendaten mit Diagnose, aktuellem Zustand des Patienten sowie Eintreffzeit.

Im Rahmen der Qualitätssicherung ist es unabdingbar, die für die Klinik erstellten Algorithmen regelmäßig zu überprüfen. Insbesondere Veränderungen von Erreichbarkeiten wie neue Telefonnummern können fatale Folgen haben. Im Universitätsklinikum Augsburg finden daher neben den regelmäßigen Schockraumkonferenzen, bei denen Einzelfälle besprochen werden, halbjährlich Kon- 
ferenzen der „Arbeitsgruppe Schockraum“ statt. Im gleichen Abstand muss von den Schockraumverantwortlichen aller Abteilungen die Telefonliste auf Richtigkeit überprüft und aktualisiert werden.

\section{Schockraumteam - \\ Basisschockraumteam}

Gemäß den Vorgaben der Deutschen Gesellschaft für Unfallchirurgie unterscheidet man zwischen Basisschockraumteam und erweitertem Schockraumteam [1].

Das Basisteam muss sofort 24 Stunden an 365 Tagen verfügbar sein und wird im überregionalen Traumazentrum gebildet von:

- 1 Facharzt (FA) bzw. Weiterbildungsassistent für Orthopädie und Unfallchirurgie

- 1 Weiterbildungsassistent in Orthopädie und Unfallchirurgie bzw.

- 1 Weiterbildungsassistent in Zusatzweiterbildung Spezielle Unfallchirurgie bzw.

- 1 Weiterbildungsassistent in Viszeralchirurgie oder Allgemeinchirurgie

- 1 Facharzt für Anästhesiologie bzw. Weiterbildungsassistent (FA-Standard)

- 1 Facharzt für Radiologie bzw. Weiterbildungsassistent (FA-Standard)

- 2 Pflegekräfte Chirurgie

- 1 Pflegekraft Anästhesiologie

- 1 medizinisch-technische Radiologiefachkraft (MTRA)

- Transportpersonal

Das erweiterte Schockraumteam muss innerhalb von 2030 Minuten an 365 Tagen verfügbar sein und wird im überregionalen Traumazentrum gebildet von:

- Facharzt für Orthopädie und Unfallchirurgie mit Zusatzweiterbildungsqualifikation Spezielle Unfallchirurgie oder FA für Chirurgie mit der Zusatbezeichnung „Spezielle Unfallchirurgie“ Unfallchirurgie (Oberarzt)

- Facharzt für Viszeralchirurgie oder Allgemeinchirurgie (Oberarzt)

- Facharzt für Anästhesiologie (Oberarzt)

- Facharzt für Neurochirurgie (Oberarzt)

- Facharzt für Radiologie (Oberarzt) mit Kenntnissen in interventioneller Radiologie

- Facharzt für Gefäßchirurgie

- Facharzt für Herz- und/oder Thoraxchirurgie

- Facharzt für Mund-, Kiefer-, Gesichtschirurgie

- Facharzt für HNO

- Facharzt für Augenheilkunde

- Facharzt für Urologie

- 2 OP-Pflegekräfte

- weitere Rufdienste zur gleichzeitigen Versorgung mehrerer Schwerverletzter

fakultativ - überregionales Traumazentrum

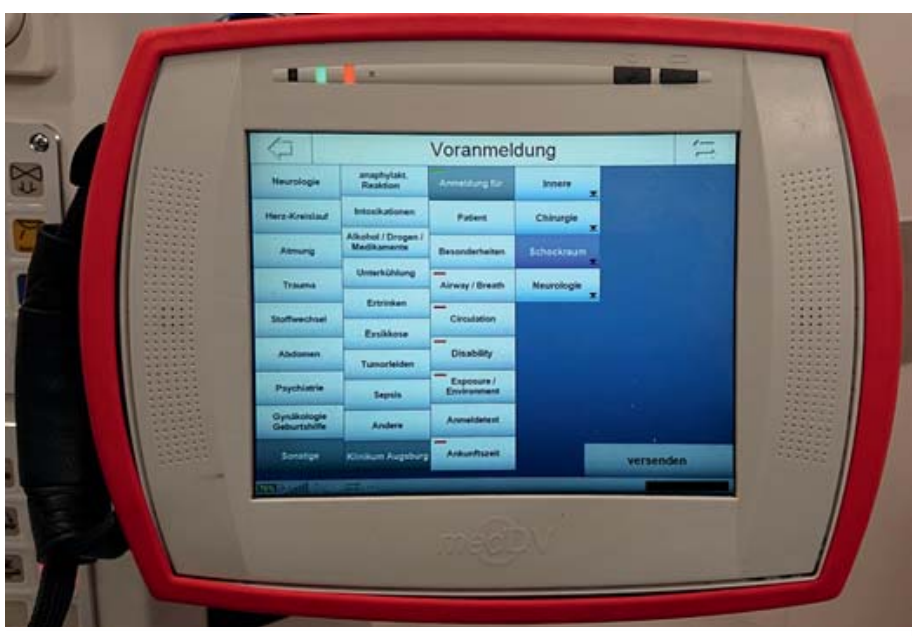

Abb. 5 NIDA-PAD.

- Facharzt für Gynäkologie

- Facharzt mit Zusatzweiterbildung Handchirurgie (Facharzt für Orthopädie und Unfallchirurgie oder Facharzt für plastische Chirurgie)

- Facharzt für Kinderchirurgie oder Pädiatrie

- 1 Pflegekraft Anästhesiologie

- 1 medizinisch-technische Radiologiefachkraft (MTRA)

- Transportpersonal

Im Universitätsklinikum Augsburg ist der Trauma-Leader der unfallchirurgische Oberarzt oder der unfallchirurgische Erstdienst mit der entsprechenden vorgeschriebenen Qualifikation. Zusätzlich zu dem genannten Basisbzw. erweiterten Schockraumteam wird bei jedem Schockraumalarm die Blutbank bzw. das Labor über das Eintreffen eines schwerstverletzten Patienten informiert.

\section{Medizinische Ausstattung der Schockräume des Universitätsklinikums Augsburg}

\section{Definition Schockraum}

Eine allgemein gültige Definition „Schockraum“ ist in der Literatur nicht zu finden. Letztendlich sollte der Schockraum ein zentraler Ort in einer Notaufnahme eines Krankenhauses sein, an dem kritisch kranke Patienten jeden Alters entsprechend diagnostiziert und erstversorgt werden können.

Der ideale Schockraum muss für die optimale Versorgung von Schwerstverletzten räumlich, apparativ, technisch und personell geeignet sein. Im Schockraum muss eine ideale Versorgung von schwerverletzten Patienten mit Sicherung oder Wiederherstellung der Vitalfunktion, einer schnellen, genauen Diagnostik und der Durchführung von Notfalleingriffen möglich sein [9]. 


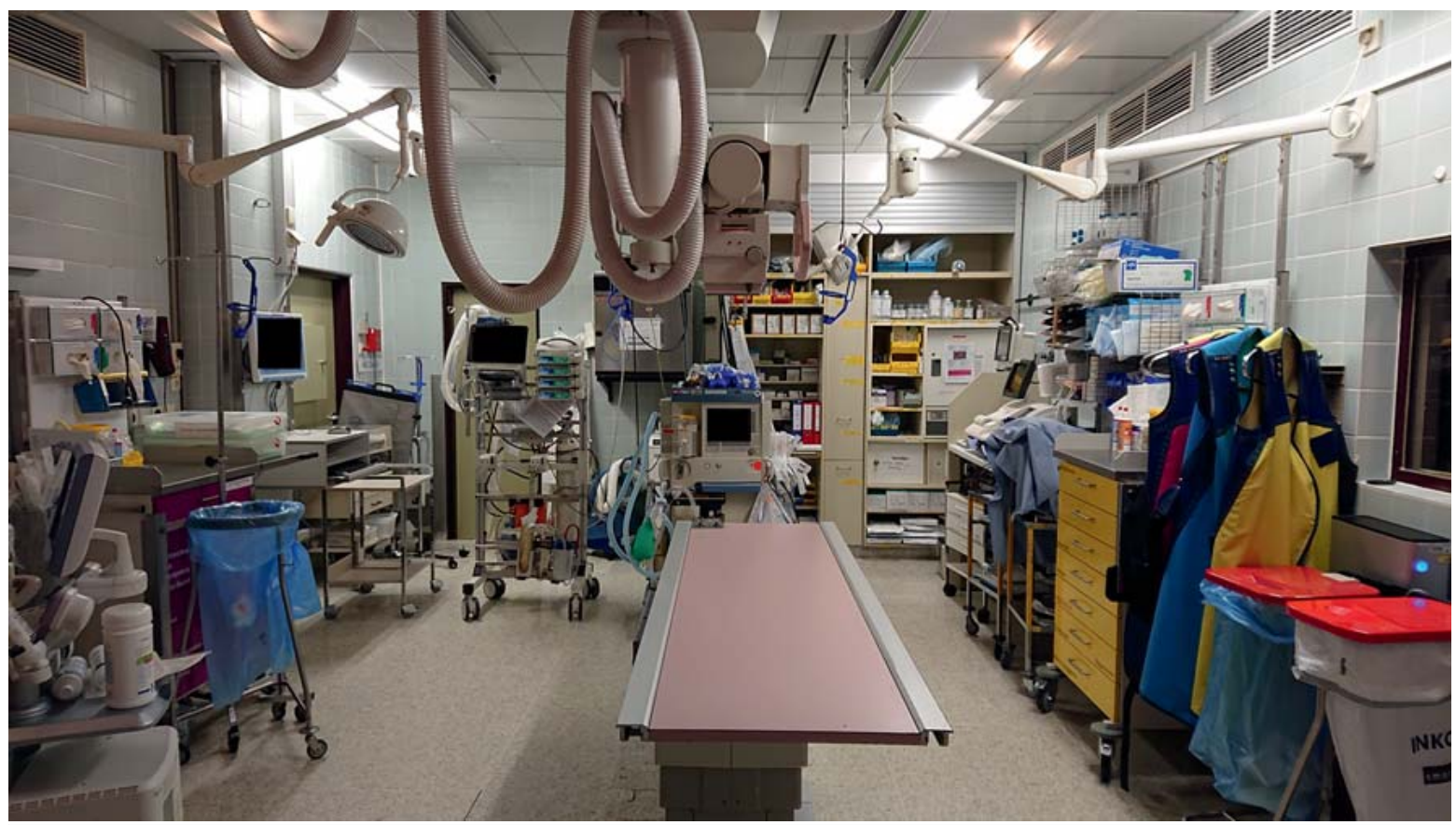

- Abb. 6 Schockraum 1.

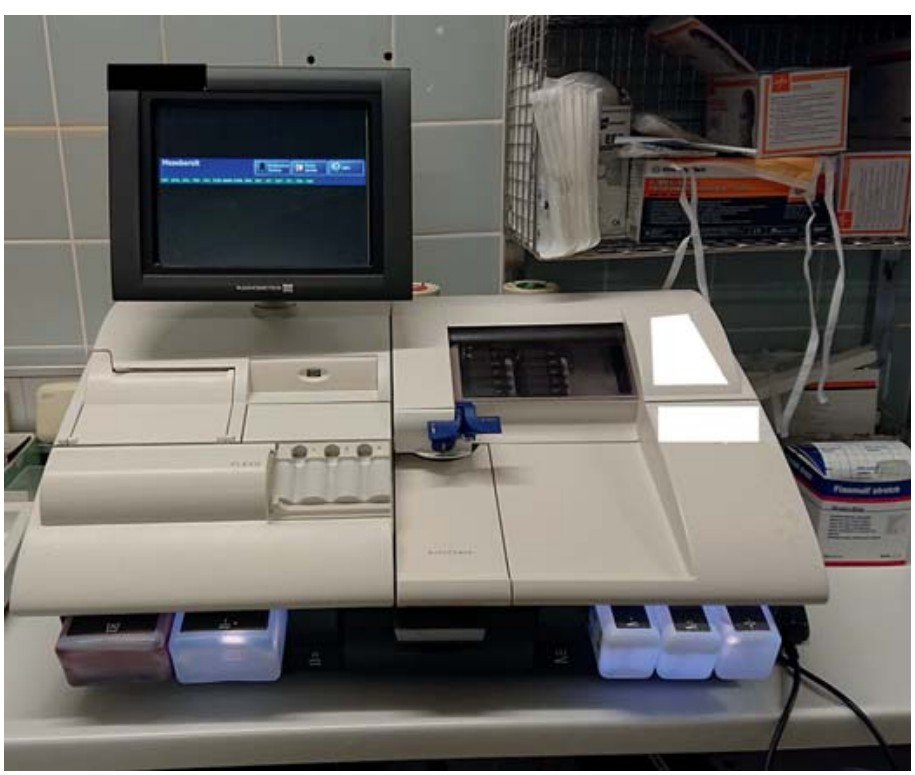

Abb. 7 Blutgasanalyse.

In einem Großkrankenhaus wird es viele solcher Behandlungsplätze geben. Jede Klinik muss für sich nach der individuellen Leistungsfähigkeit und unter Berücksichtigung der zu behandelnden Patienten ein Konzept erarbeiten.

Im Schockraum des Universitätsklinikums Augsburg werden alle Patienten entsprechend der Schockraumindikationsliste ( $\bullet$ Abb. $\mathbf{3}$ ) erstversorgt.
Unter Berücksichtigung des Weißbuchs und der AWMFS3-Leitlinie Polytrauma ist die logistische Materialvorhaltung im Schockraum in 3 Säulen zu unterteilen:

- Anästhesiearbeitsplatz

- Diagnostik

- chirurgisches Equipment

Alle 3 Säulen müssen zusammenspielen und zeitgleich abrufbar sein.

Im Hauptschockraum (Schockraum 1, > Abb. 6) werden die meisten unserer schwerstverletzten Patienten behandelt.

Ausstattung des Anästhesiearbeitsplatzes:

- Beatmungseinheit fest (Narkosegerät) (• Abb. 8)

- Monitoring fest

- 6 Perfusoren

- Wärmeschrank

- Modul schwieriger Atemweg

- Medikamentenschrank ( $>$ Abb. 9 und 10)

- Infusionen

- Druckbeutel

- Zugangsset für invasives Monitoring aller Art inkl. ZVK (zentraler Venenkatheter), Arterie, Shaldon-Katheter

- Blutgasanalyse (- Abb. 7)

- Blutabnahmeröhrchen

- Blutkultur

- Bedside-Test

- Narkoseprotokolle

- sterile Mäntel mit Handschuhen 


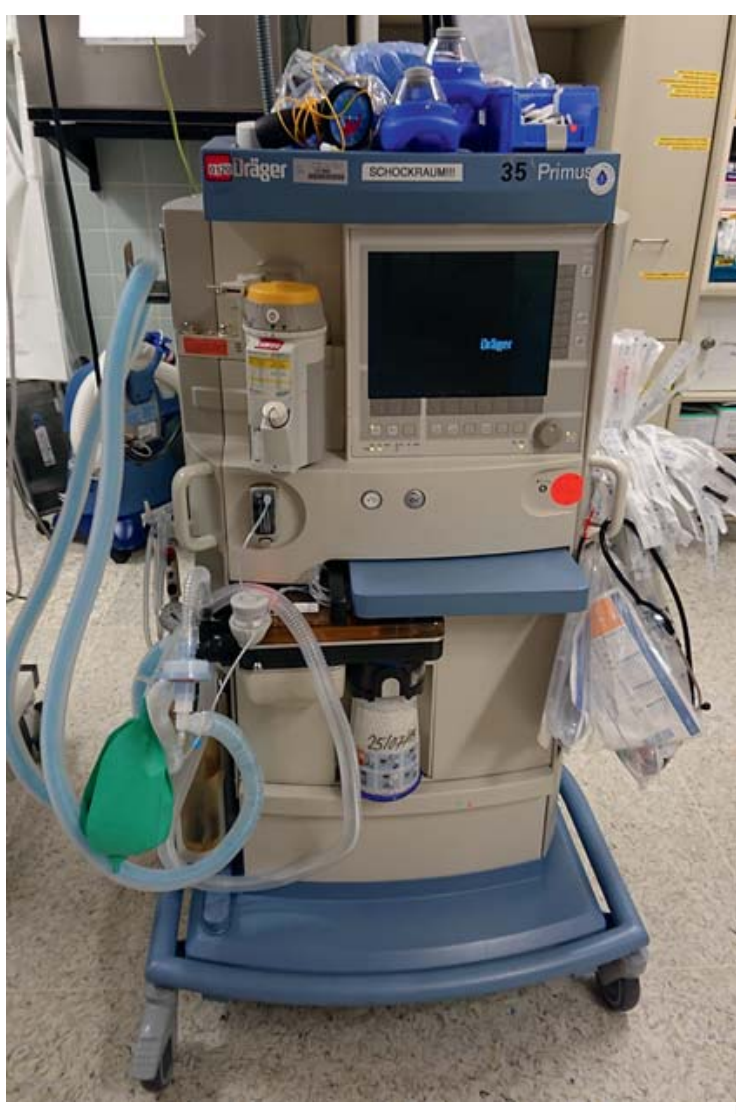

Abb. 8 Beatmung.

- 2 EDV-Arbeitsplätze

- 2 Telefone

- Beatmungseinheit mobil ( $\bullet$ Abb. 11)

- Monitoring mobil

- Schockbox (wird zum Eintreffen des Polytraumapatienten im Schockraum bereit gestellt)

- Modul Kinderanästhesie

Ausstattung des chirurgischen Arbeitsplatzes:

- 1 EDV-Arbeitsplatz

- 1 Telefon

- Beckengürtel

- Stifneck alle Größen

- Thoraxdrainagen

- chirurgische Siebe:

- Thoraxdrainage

- Thorakotomie mit Rippenspreizer

- Blutung (Gefäßklemmen)

- HNO-Wagen

- Tourniquets

- Bauchtücher

- Verbandmaterial

- Verbrennungsset

- Replantationsbeutel

- Hämostyptika

- Schockraumuhr (> Abb. 13)

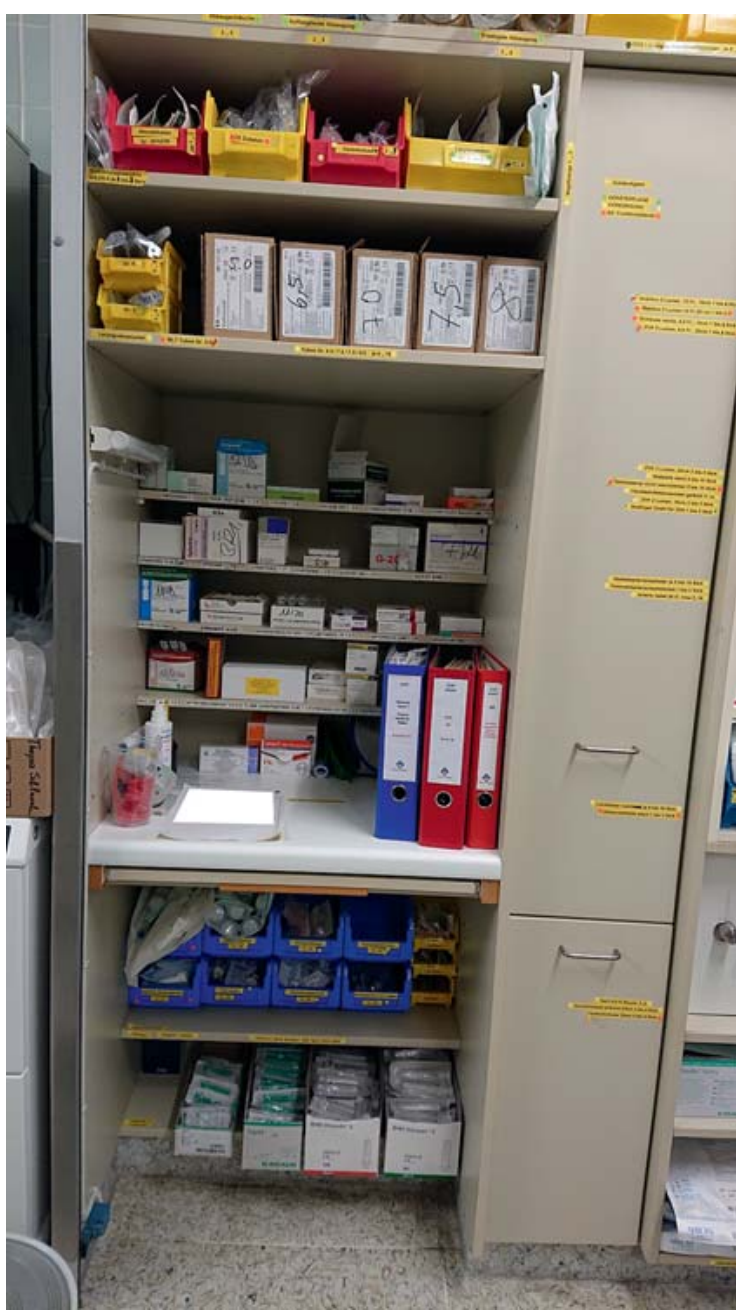

- Abb. 9 Schrank 1 Anästhesie.

- markierte Röntgenschürzen (- Abb. 12)

- Absaugeinheit

- Hochfrequenz-Chirurgie-Gerät, bipolar-monopolar

- Diagnostik/Radiologie

- Ultraschallgerät

- Röntgentisch mit Deckenstativ

außerhalb des Schockraums:

- CT ( $\triangleright$ Abb. 14), Angiografie, Intervention in unmittelbarer Nähe

- MRT

\section{Beckenzwinge}

Die Beckenzwinge ( $\triangleright$ Abb. 15) war zur Notfallbehandlung der hämodynamisch instabilen Beckenfraktur bis vor wenigen Jahren „State of the Art“ und wurde in fast allen Schockräumen überregionaler und regionaler Traumazentren vorgehalten. Durch die Volumenzunahme des Beckens bei instabilitätsbedingter Fehlstellung durch Frakturen und/oder Verletzung der Symphyse bzw. der 


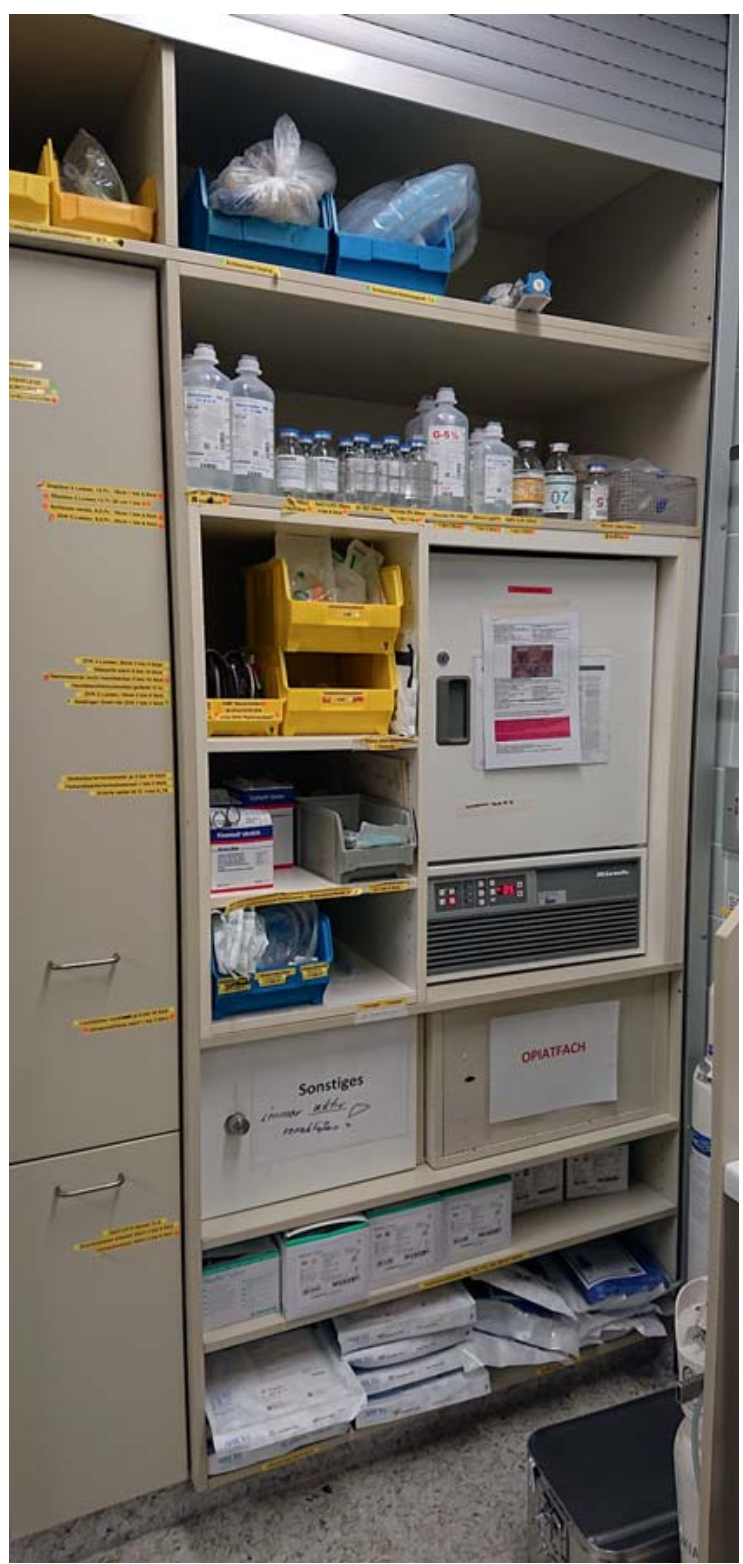

- Abb. 10 Schrank 2 Anästhesie.

Iliosakralgelenkfugen kommt es zu massiven Blutungen meist aus dem präsakralen Venenplexus. Es gab die Empfehlung entsprechend der Leitlinie der DGU, mit der Beckenzwinge schnellstmöglich das knöcherne Becken am hinteren Beckenring zu stabilisieren, um eine Minimierung des intrapelvinen Volumens zu erreichen, sodass eine Selbsttamponade der Blutung möglich wird [10].

Mit Einführung des Beckengurtes (pelvic binder) konnte in zahlreichen Studien die Effektivität dieser nicht invasiven Maßnahme bei kreislaufinstabilen Beckenringverletzungen gezeigt werden [11-13].

Die Anlage des Beckengurtes ist einfach und effektiv, wohingegen die Anlage einer Beckenzwinge schwierig sein

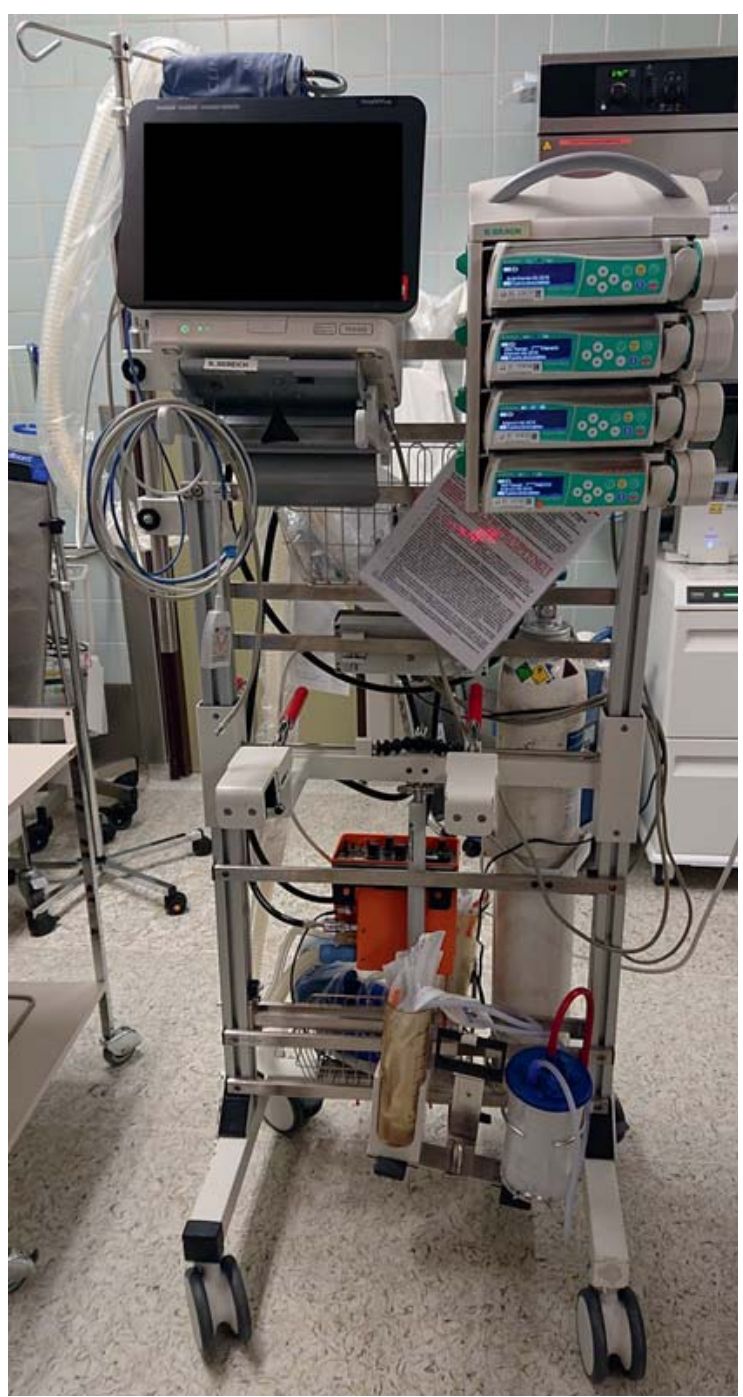

Abb. 11 Kralle.

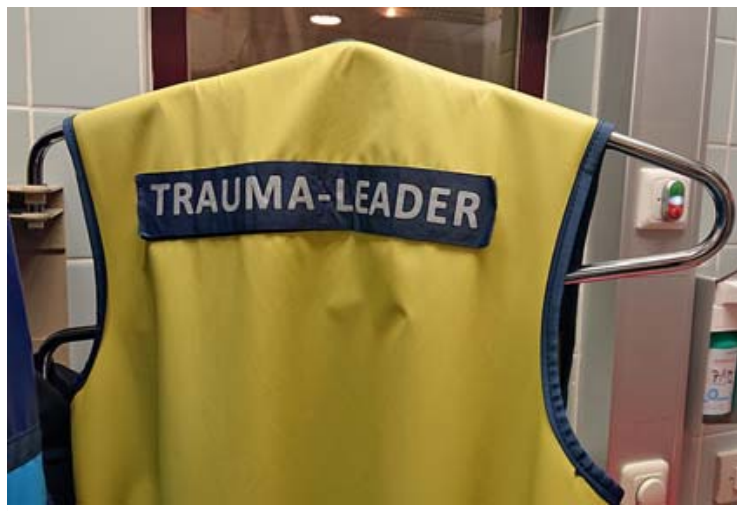

- Abb. 12 Röntgenschürze mit Markierung. 
kann. Insbesondere ohne Bildwandler stellt die korrekte Anlage einer Beckenzwinge eine Herausforderung dar. Zwischenzeitlich haben wir im Schockraum keine Beckenzwinge mehr. Unser Beckenalgorithmus sieht nun die Anlage des Pelvic Binders mit anschließender Diagnostik bestehend aus FAST-Sonografie, Röntgen des Beckens sowie CT mit Kontrastmittel vor. Ist eine Stabilisierung notwendig, wird diese im OP durchgeführt. Dort kommt die Beckenzwinge zur Stabilisierung des hinteren Beckenringes mit additivem Fixateur externe ventral sowie, wenn notwendig, pelvines Packing zum Einsatz [14].

\section{Chirurgische Intervention/ Operation im Schockraum}

Die Schockraumphase bei der Versorgung schwerstverletzter Patientin dient dazu, möglichst alle Verletzungen, insbesondere die lebensbedrohlichen, rasch zu detektieren und möglichst schnell der entsprechenden Therapie zuzuführen.

Operationen, selbst Notoperationen entsprechend dem S3-Leitlinien-Abschnitt „Erste OP-Phase“, sollten nicht im Schockraum, sondern im OP durchgeführt werden. Verschiedene logistische Fakten führen zu dieser Empfehlung:

- Die Schockraumphase sollte so kurz wie möglich und so lange wie notwendig dauern. Das Ziel bleibt das Erfassen der lebensbedrohlichen Verletzungen und die Erststabilisierung des Patienten.

- Der Schockraum und das Schockraumteam sollten schnellstmöglich für einen weiteren Schwerverletzten wieder frei werden.

- Die Vorhaltung von OP-Sieben, welche für die Durchführung von Operationen gemäß der „Ersten OPPhase“ notwendig sind, ist je nach baulichen Strukturen im Bereich der Notaufnahme und Radiologie nicht möglich.

- Der Weg vom Schockraum in den OP ist meist kurz. Ein Zeitverlust daher nicht relevant.

- Für die Notfalloperation steht im OP alles zur Verfügung und muss nicht erst herbeigebracht werden.

Die operativen chirurgischen Maßnahmen im Schockraum im Universitätsklinikum Augsburg beschränken sich daher auf folgende Eingriffe:

- Anlage Thoraxdrainage über Minithorakotomie

- Gefäßklemme bei sichtbarer Blutungsquelle

- Tamponaden (offene Höhlenverletzung, Blutung HNO, Gynäkologie)

- Entlasten einer Perikardtamponade

- erweiterte Thorakotomie im Rahmen einer Reanimation bei penetrierenden Thoraxverletzungen und Eintritt der Reanimationspflichtigkeit im Schockraum

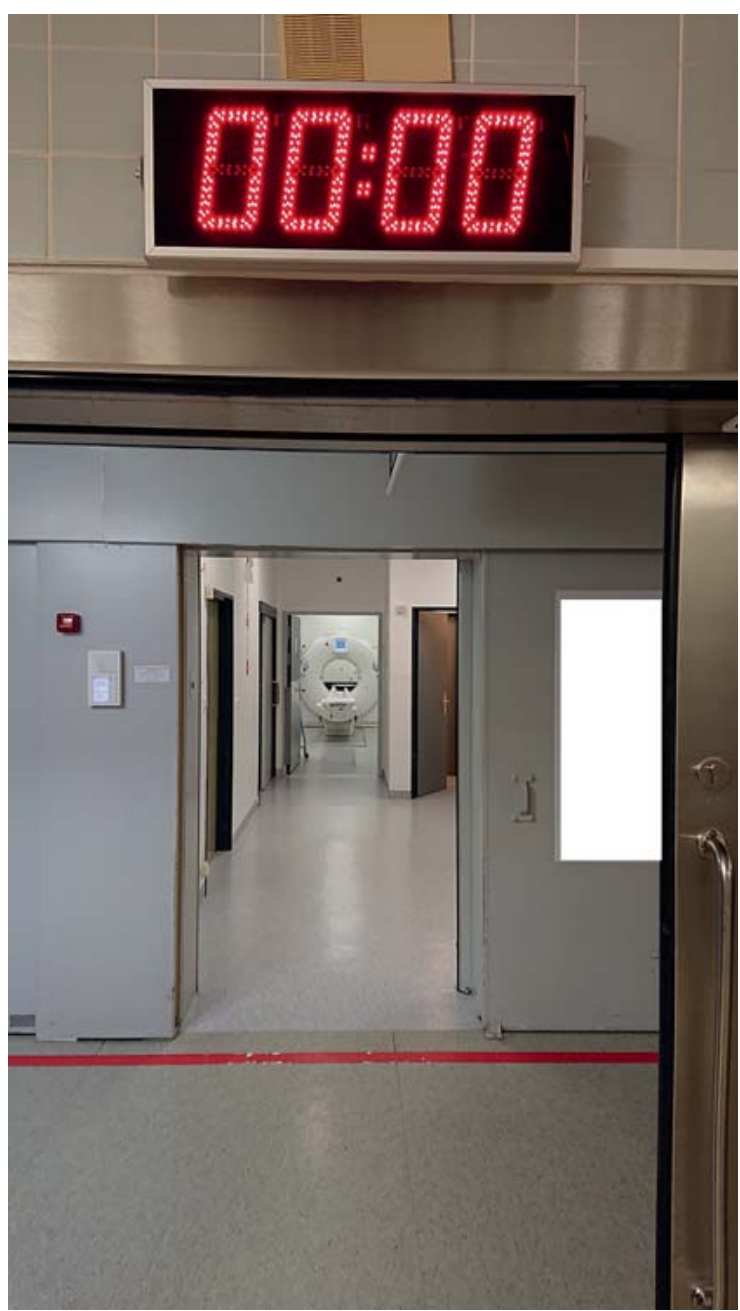

Abb. 13 Schockraumuhr.

\section{Unterscheidung Schockraumteam, medizinische Ausstattung und chirurgische Intervention in lokalen und regionalen Traumazentren}

\section{Schockraumteam lokales und regionales Traumazentrum}

Das Basisteam Schockraum eines lokalen Traumazentrums verfügt nicht von Beginn an über einen Radiologen. Dieser ist nach Vorgaben des Weißbuchs Schwerverletztenversorgung der DGU erst im erweiterten Schockraumteam nach 20-30 Minuten vor Ort [1]. Das Basisschockraumteam im regionalen Traumazentrum (TZ) unterscheidet sich vom überregionalen Traumazentrum nicht. 


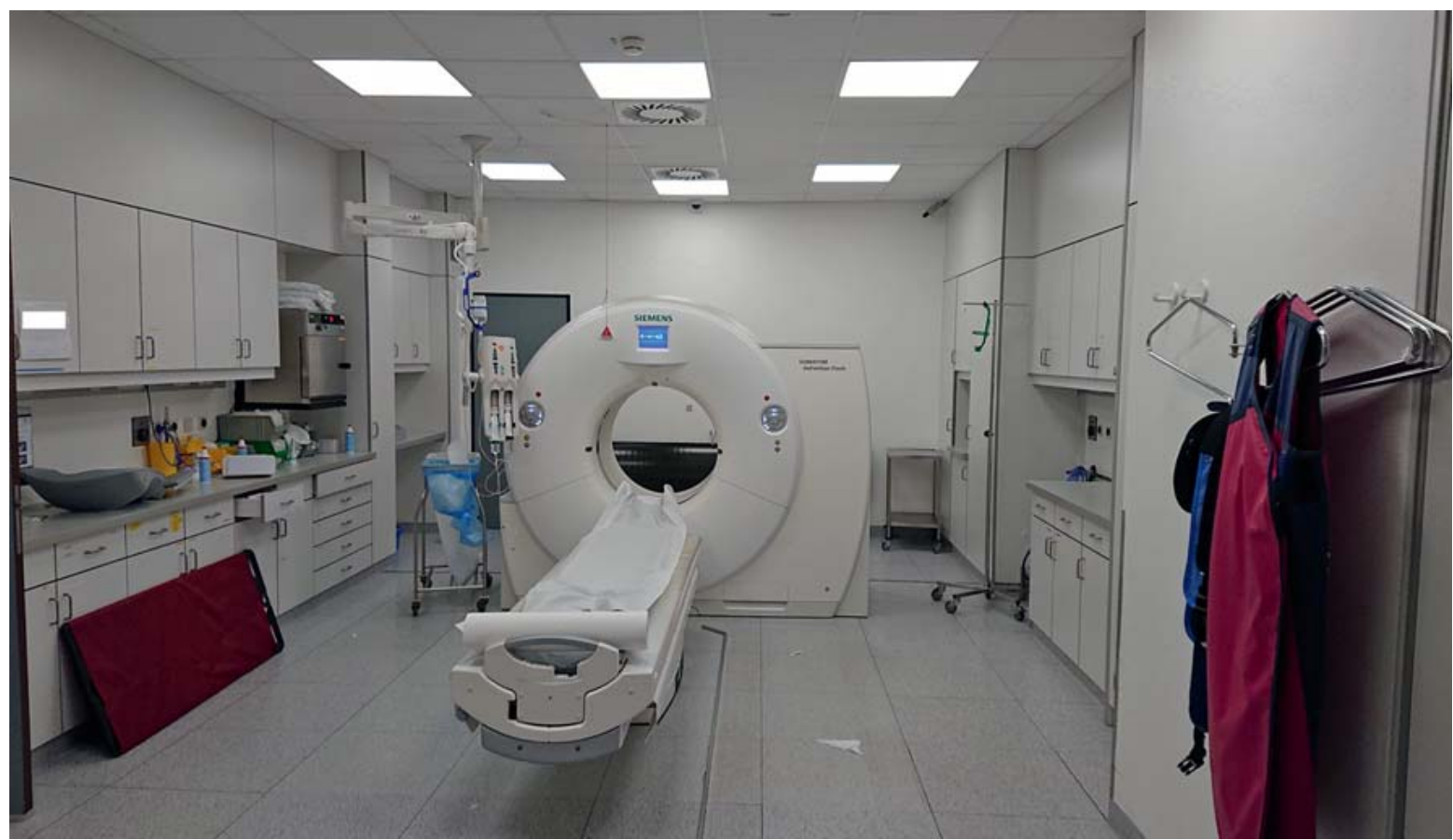

- Abb. 14 Schockraum-CT.

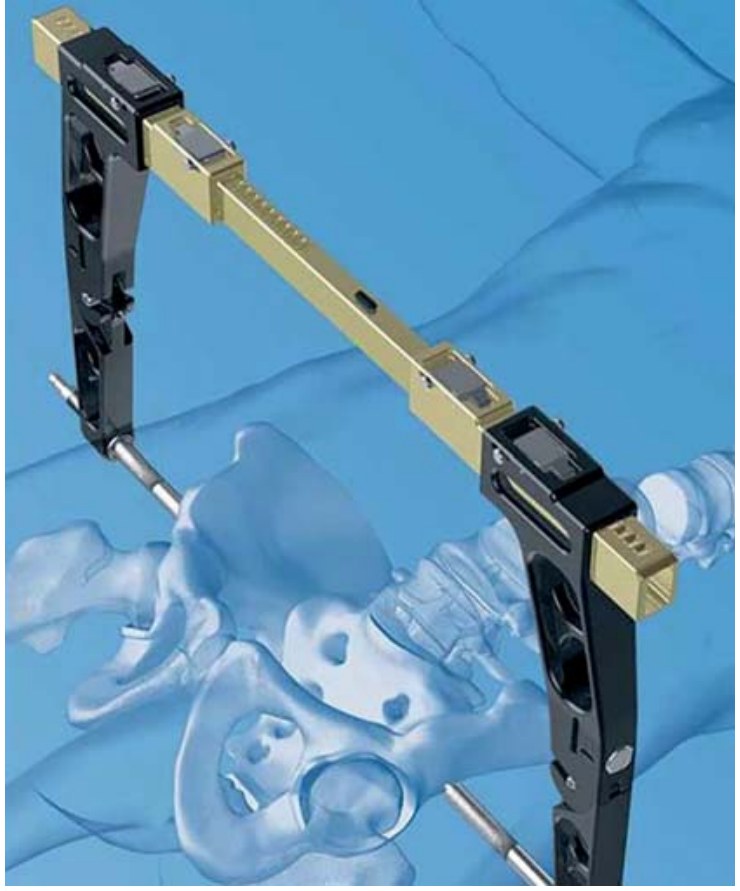

- Abb. 15 Beckenzwinge. Quelle: OP-Technik, 05/2008, Synthes Inc. oder deren Tochtergesellschaften.

\section{Medizinische Ausstattung im Schockraum „lokales Traumazentrum“}

Die Aufgabe einer Klinik mit Status lokales TZ ist wie folgt definiert: „Lokale Traumazentren haben ihre wesentliche Funktion in der flächendeckenden Versorgung der häufigsten Einzelverletzungen. Für die Schwerverletzten-Versorgung, insbesondere außerhalb von Ballungszentren, dienen sie als initiale Anlaufstellen mit der wichtigen Aufgabe einer adäquaten Erstbehandlung und der zielgerichteten Weiterleitung, wenn ein primärer zeitgerechter Transport in ein regionales oder überregionales Traumazentrum nicht möglich ist (Aufnahmeverpflichtung)“ [1].

Die Vorhaltung eines Computertomografen ist nicht zwingend notwendig. Letztendlich sollte jedoch auch ein lokales Traumazentrum über die gleiche medizinische Ausstattung im Schockraum verfügen wie oben beschrieben.

\section{Medizinische Ausstattung im Schockraum „regionales Traumazentrum“}

Die Aufgabe einer Klinik mit Status regionales TZ ist wie folgt definiert: „Die regionalen Traumazentren haben ihre Aufgabe in der umfassenden Notfall- und Definitiv-Versorgung von Schwerverletzten unter Vorhaltung ausreichender Intensiv- und Operationskapazitäten. Die apparativen und personellen Ressourcen umfassen die jederzeit verfügbare Facharztkompetenz mit Weiterbildung in der Speziellen Unfallchirurgie sowie auch regelhaft den Einbezug weiterer Fachdisziplinen (z.B. Neurochirurgie) und eine diesem Versorgungsniveau angepasste diagnostische und therapeutisch-operative Ausstattung, Beteiligung am Ka- 
tastrophenschutz mit Bereitstellung von Behandlungskapazitäten. Es unterscheidet sich vom überregionalen Traumazentrum durch eingeschränkte Kapazität (1 Schockraumbehandlungsplatz) und durch eingeschränkte Kompetenz in der Behandlung besonders komplexer Verletzungen (z. B. thorakale Aortenverletzungen).“

Die sehr hohen Anforderungen an regionale Traumazentren erfordern die technische Schockraumausstattung eines überregionalen Traumazentrums.

\section{Fazit}

Die Versorgung von Schwerstverletzten ist sowohl eine medizinische als auch eine logistische und sozioökonomische Herausforderung [15]. Der Personalbedarf an hochqualifizierten Medizinern und Pflegekräften ist erheblich. Der finanzielle Aufwand für die an der Polytraumaversorgung teilnehmenden Kliniken ist nicht zuletzt auch wegen der notwendigen Zusatzqualifikationen des Personals hoch. Die Versorgungsstrategie bei der Polytraumaversorgung hing bisher häufig mehr von der eigenen Erfahrung des behandelnden Arztes als von der tatsächlich vorhandenen Evidenz ab [16]. Die Empfehlungen aus der AWMF-S3-Leitlinie Polytrauma/Schwerverletztenbehandlung müssen in den Schockraumalgorithmus integriert werden und bisherige Behandlungsstrategien ggf. verlassen werden.

\section{Interessenkonflikt}

Die Autoren geben an, dass kein Interessenkonflikt besteht.

Autorinnen/Autoren

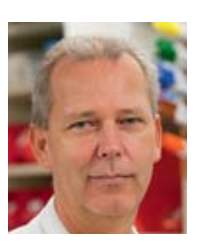

\section{Michael Ecker}

Dr. med., Leitender Oberarzt, Klinik für Unfallchirurgie, Orthopädie, Plastische und Handchirurgie, Universitätsklinikum Augsburg

\section{Korrespondenzadresse}

\author{
Dr. Michael Ecker \\ Klinik für Unfallchirurgie, Orthopädie, Plastische \\ und Handchirurgie \\ Universitätsklinikum Augsburg \\ Stenglinstraße 2 \\ 86156 Augsburg \\ Tel.: 0821/4002936 \\ Fax: $0821 / 4003313$ \\ michael.ecker@uk-augsburg.de
}

Literatur

[1] Deutsche Gesellschaft für Unfallchirurgie. Weißbuch Schwerverletztenversorgung. Im Internet: https://www.dgu-online. de/fileadmin/published_content/5.Qualitaet_und_Sicherheit/
PDF/20_07_2012_Weissbuch_Schwerverletztenversorgung_ Auflage2.pdf; Stand: 30.10.2019

[2] Sektion Intensiv- \& Notfallmedizin, Schwerverletztenversorgung der Deutschen Gesellschaft für Unfallchirurgie e.V.; AUC - Akademie der Unfallchirurgie GmbH. TraumaRegister DGU. Jahresbericht 2018. Im Internet: http://www.traumaregister-dgu.de/fileadmin/user_upload/traumaregister-dgu. de/docs/Downloads/TR-DGU-Jahresbericht_2018.pdf; Stand: 30.10.2019

[3] AWMF. S3-Leitlinie Polytrauma/Schwerverletzten-Behandlung, Register-Nr.012/019. Im Internet: https://www.awmf.org/ uploads/tx_szleitlinien/012-019|_S3_Polytrauma_Schwerverletzten-Behandlung_2017-08.pdf; Stand: 30.10.2019

[4] Bouillon B, Kanz KG, Lackner CK et al. Die Bedeutung des Advanced Trauma Life Support (ATLS) im Schockraum. Unfallchirurg 2004; 107: 844-850

[5] Wurmb T, Balling H, Frühwald $\mathrm{P}$ et al. Polytraumamanagement im Wandel. Zeitanalyse neuer Strategien für die Schockraumversorgung. Unfallchirurg 2009; 112: 390-399

[6] Boschin M, Vordemvenne T. [First aid and management of multiple trauma: in-hospital trauma care]. Anästhesiol Intensivmed Notfallmed Schmerzther 2012; 47: 716-723. doi:10.1055/s-0032-1331369

[7] Flintrop J. Ökonomisierung im Krankenhaus: Zwischen Verantwortung und Profitstreben. Im Internet: https://www.aerzteblatt.de/nachrichten/71543/Oekonomisierung-im-Krankenhaus-Zwischen-Verantwortung-und-Profitstreben; Stand: 30.10.2019

[8] Bauer C. Projekt „NIDA“. Im Internet: https://www.aerztezeitung.de/praxis_wirtschaft/e-health/article/972936/notfalltelemedizin-projekt-nida.html; Stand: 30.10.2019

[9] Grasslober M. Der ideale Schockraum. Eur Surg 2002; 34 (Suppl. 182): S40-S42

[10] Tiemann AH, Hofmann GO. Beckenzwinge. Notfallbehandlung der (hämodynamisch) instabilen Beckenfraktur. Trauma Berufskrankh 2008. doi:10.1007/s10039-008-1386-9

[11] Wohlrath B, Trentzsch H, Hoffmann R et al. Präklinische und klinische Versorgung der instabilen Beckenverletzung. Unfallchirurg 2016; 119: 755-762

[12] Esmer E, Esmer E, Derst P et al. Einfluss der externen Beckenstabilisierung bei hämodynamisch instabilen Beckenfrakturen. Unfallchirurg 2017; 120: 312-319

[13] Schweigkofler U, Wohlrath B, Paffrath T et al. „Clear-thePelvis-Algorithmus“: Handlungsempfehlung zur Freigabe des Beckens nach nicht invasiver Stabilisierung mittels Beckengurt im Rahmen der Schockraumversorgung. Z Orthop Unfall 2016; 154: 470-476

[14] Fritz T, Pohlemann T. Beckenchirurgie - Akutversorgung. OPJournal 2018; 34: 26-32

[15] Frink M, Lechler P, Debus F et al. Polytrauma und Schockraummanagement. Dtsch Ärztebl Int 2017; 114: 497-503

[16] Rixen D, Steinhausen E, Dahmen J et al. S3-Leitlinie Polytrau$\mathrm{ma} /$ Schwerverletzten-Behandlung. Erste Operationsphase: Bedeutung - Möglichkeiten - Schwierigkeiten? Unfallchirurg 2012; 115: 22-29. doi:10.1007/s00113-011-2104-9

Bibliografie

DOI https://doi.org/10.1055/a-0990-2053

online publiziert 03.03.2020 | OP-JOURNAL 2020; 36: 50-59

(c) Georg Thieme Verlag KG Stuttgart · New York

ISSN 0178-1715 